\title{
Artificial neural network modeling of the water quality index using land use areas as predictors
}

\begin{abstract}
This paper describes the design of an artificial neural network (ANN) model to predict the water quality index (WQI) using land use areas as predictors. Ten-year records of land use statistics and water quality data for Kinta River (Malaysia) were employed in the modeling process. The most accurate WQI predictions were obtained with the network architecture 723-1; the back propagation training algorithm; and a learning rate of 0.02 . The WQI forecasts of this model had significant ( $p<0.01)$, positive, very high correlation $(\rho s=0.882)$ with the measured WQI values. Sensitivity analysis revealed that the relative importance of the land use classes to WQI predictions followed the order: mining > rubber > forest > logging > urban areas $>$ agriculture $>$ oil palm. These findings show that the ANNs are highly reliable means of relating water quality to land use, thus integrating land use development with river water quality management.
\end{abstract}

Keyword: Artificial neural network; Function approximation; Land use areas; Threelayer perceptron; Unweighted harmonic square mean; Water quality index; Weighted arithmetic mean 